\title{
Plant NIGT1/HRS1/HHO Transcription Factors: Key Regulators with Multiple Roles in Plant Growth, Development, and Stress Responses
}

\author{
Qian $\mathrm{Li}^{1,2}$, Luyan Zhou ${ }^{1,2}$, Yuhong $\mathrm{Li}^{3}$, Dongping Zhang ${ }^{1,2}$ and Yong Gao ${ }^{1,2, *(\mathbb{D}}$ \\ 1 Jiangsu Key Laboratory of Crop Genomics and Molecular Breeding/Key Laboratory of Plant Functional \\ Genomics of the Ministry of Education, Yangzhou University, Yangzhou 225009, China; \\ LiQianyzu@163.com (Q.L.); zhouluyan1997@163.com (L.Z.); dpzhang@yzu.edu.cn (D.Z.) \\ 2 Co-Innovation Center for Modern Production Technology of Grain Crops of Jiangsu Province, Joint \\ International Research Laboratory of Agriculture and Agri-Product Safety of the Ministry of Education, \\ Yangzhou University, Yangzhou 225009, China \\ 3 Institute of Agricultural Sciences for Lixiahe Region in Jiangsu, Yangzhou 225009, China; yhlirice@163.com \\ * Correspondence: gaoyong@yzu.edu.cn; Tel.: +86-0514-87997217
}

Citation: Li, Q.; Zhou, L.; Li, Y.;

Zhang, D.; Gao, Y. Plant

NIGT1/HRS1/HHO Transcription

Factors: Key Regulators with

Multiple Roles in Plant Growth,

Development, and Stress Responses.

Int. J. Mol. Sci. 2021, 22, 8685.

https://doi.org/10.3390/ijms22168685

Academic Editor: Yong-Hwan Moon

Received: 29 July 2021

Accepted: 10 August 2021

Published: 12 August 2021

Publisher's Note: MDPI stays neutral with regard to jurisdictional claims in published maps and institutional affiliations.

Copyright: () 2021 by the authors. Licensee MDPI, Basel, Switzerland. This article is an open access article distributed under the terms and conditions of the Creative Commons Attribution (CC BY) license (https:// creativecommons.org/licenses/by/ $4.0 /)$.

\begin{abstract}
The NIGT1/HRS1/HHO transcription factor (TF) family is a new subfamily of the G2-like TF family in the GARP superfamily and contains two conserved domains: the Myb-DNA binding domain and the hydrophobic and globular domain. Some studies showed that NIGT1/HRS1/HHO TFs are involved in coordinating the absorption and utilization of nitrogen and phosphorus. NIGT1/HRS1/ HHO TFs also play an important role in plant growth and development and in the responses to abiotic stresses. This review focuses on recent advances in the structural characteristics of the NIGT1/HRS1/ HHO TF family and discusses how the roles and functions of the NIGT1/HRS1/HHO TFs operate in terms of in plant growth, development, and stress responses.
\end{abstract}

Keywords: NIGT1/HRS1/HHO transcription factors; G2-like; growth and development; abiotic stress; structure and function

\section{Introduction}

The GARP transcription factor (TF) superfamily is commonly found in plants. It is named for the Golden 2 (G2) protein in maize, the type B authentic response regulator (ARR-B) protein in Arabidopsis thaliana, and the phosphate starvation response 1 (PSR1) protein in Chlamydomonas. The GARP superfamily contains two types of TFs, ARR-B and G2-like TFs. Among them, the ARR-B TFs comprise the multi-functional domain B motif, which is related to nuclear localization and DNA binding. G2-like TFs have an Myb-DNA binding domain (Myb-DBD), which is highly similar to the B motif [1-3]. G2-like TFs are also known as Golden2-like and GLK and are derived from the G2 protein in maize [4,5]. G2-Like TFs have been found and characterized in monocots and dicots. Studies have shown that G2-Like TFs play an important role in chloroplast development, fruit quality, biotic and abiotic stress, plant senescence, and hormones.

NIGT1/HRS1/HHO TFs belong to a subfamily of the G2-Like TFs and were first identified and named in 2009 [6]. With advances in high-throughput sequencing technologies, more and more NIGT1/HRS1/HHO TF genes have been identified from $A$. thaliana, rice, and other species. Subsequently, the functions of NIGT1/HRS1/HHO TFs have been gradually found in nitrogen $(\mathrm{N})$ and phosphorus $(\mathrm{P})$ utilization, plant growth and development, and abiotic stress [7-12]. Here, the review focuses on recent advances in the structural characteristics and biological functions of the NIGT1/HRS1/HHO TFs. 


\section{Discovery and Naming of NIGT1/HRS1/HHO TF Family in Plants}

In 2009, Liu et al. [6] first reported that the G2-like TF gene At1g13300 named HRS1 exhibited hypersensitivity to the shortening of primary roots induced by low phosphate (Pi) in A. thaliana. Further study found that HRS1 has six homologous genes in the $A$. thaliana (i.e., At1g25550, At1g49560, At1g68670, At2g03500, At3g25790, and At4g37180), and these genes were named as HHO1 (HRS1 Homolog 1) to HHO6. This family was then named the HRS1/HHO TF family. In 2013, a nitrate-induced GARP transcription repressor gene NIGT1 (Os02g0325600) was reported to belong to the HRS1/HHO family in rice. A total of four homologous genes of NIGT1 (i.e., Os01g0176700, Os03g0764600, Os07g0119300, and Os12g0586300) were also found in rice. Meanwhile, the study showed that HRS1, HHO1, $\mathrm{HHO}$, and HHO3 in A. thaliana had homology to NIGT1 in rice, and all of their expressions were strongly induced by nitrate [7]. Subsequently, HRS1 and HHO1-HHO5 were also named NIGT1.4, NIGT1.3, NIGT1.2, NIGT1.1, EFM, and UIF1, respectively [8-10]. Recently, four NIGT1 homologous genes were also named OsHHO1-OsHHO4 in rice [11]. Since then, there are two naming methods for the HRS1/HHO family in A. thaliana and rice (Figure 1). The genes of the HRS1/HHO TF family have been found in other species. The HRS1/HHO family genes, VaAQUILO $(\mathrm{VaAQ})$ in V. amurensis and VvAQUILO $(V v A Q)$ in $V$. vinifera, were first reported in 2018 [12]. However, other homologous genes of $V a A Q$ and $V v A Q$ have not been found in $V$. amurensis and $V$. vinifera. In summary, in accordance with the naming and unity principles, seven members of this family were named HRS1 and HHO1-HHO6 in A. thaliana, and five members were named OsNIGT1 and OsHHO1-OsHHO4 in rice. Finally, this family was called the NIGT1/HRS1/HHO TF family.

A

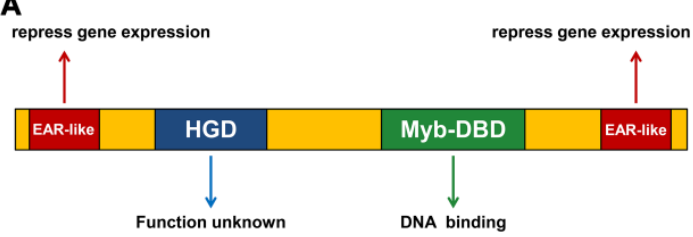

C
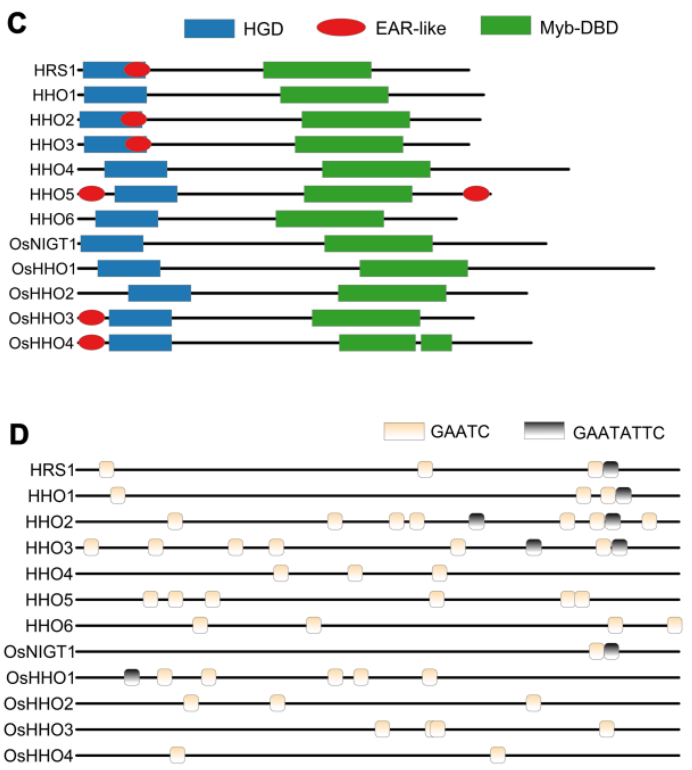

B
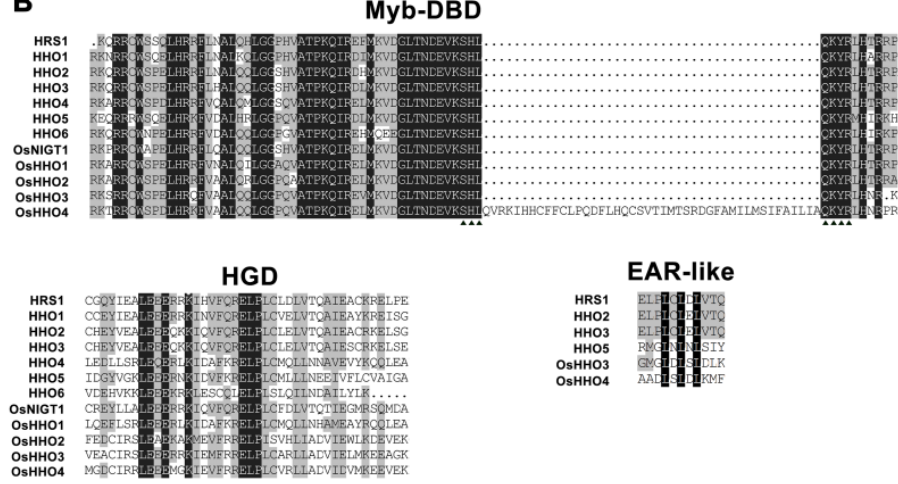

E

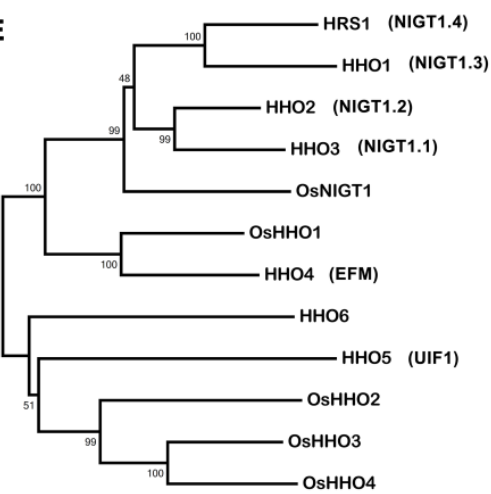

At1g13300

At3g25790

At1g68670

At2g25550

Os02g0325600

Os01g0176700

At2g03500

At1949560

At4g 37180

Os12g0586300

Os03g0764600

Os07g0119300

Figure 1. Classification and structure of the NIGT1/HRS1/HHO family. (A) The structural model of the NIGT1/HRS1/HHO family consisting of three types of conserved domains: Myb-DBD (green), HGD (blue), and EAR like (red). (B) The sequence alignment of the conserved domains of the NIGT1/HRS1/HHO family. Fully conserved amino acids are shaded in black, 
and similar amino acids ( $>50 \%$ identical) are shaded in gray. At the top, the conserved Myb-DBD domain contains an HLH motif and an SHLQKYR sequence tagged with a triangle. There is the conserved C-terminal hydrophobic domain HGD at the bottom left. The bottom right partially contains an EAR-like motif (LxLxL, where L is leucine, and X is any amino acid). (C) A schematic diagram showing the domains of various NIGT1/HRS1/HHO TFs in A. thaliana and rice. (D) There are two types of cis-regulatory motifs, GAATC and GAATATTC, in the $2500 \mathrm{bp}$ promoter region of various NIGT1/HRS1/HHO TFs in A. thaliana and rice. (E) The phylogenetic tree of the NIGT1/HRS1/HHO family constructed on the basis of the neighbor-joining method, and the gene IDs and names of each gene are displayed.

\section{Structure of NIGT1/HRS1/HHO TF Family}

NIGT1/HRS1/HHO TFs belong to a subfamily of G2-like TFs. All G2-like TFs have highly conserved Myb-DBD, and this domain contains an HLH (Helix-Loop-Helix) motif. The HLH motif can bind to DNA to regulate various physiological processes and can participate in the dimerization of transcriptional regulators. In an HLH motif, the first helix consists of 14 amino acids starting from PELHRR and its variant motif. The second helix starts from NI/VASHLQ and its variant motifs and extends to different lengths in various G2-Like proteins. Between the two helices is a loop containing 22 amino acids $[13,14]$. The $\mathrm{C}$ terminal of Myb-DBD contains a conserved SHLQ(K/M) (Y/F) R motif [3]. Studies have also shown that some G2-like proteins have a GLK/C-terminal box (GCT box) located in the C-terminal part. This GCT box is encoded by the last exon and plays a role in the dimerization process [14] (Figure 1A,B).

In addition to Myb-DBD, NIGT1/HRS1/HHO TFs also have a conserved hydrophobic and globular domain (HGD), which is rich in hydrophobic amino acids at the $\mathrm{N}$ terminal. Up until now, the function of HGD has not yet been studied. Moreover, some NIGT1/HRS1/HHO TFs also contain one or two different EAR-like motifs at their N or $\mathrm{C}$ terminal [9]. EAR-like motifs play an important role in inhibiting gene expression as transcription repressors or recruit corepressors [15,16] (Figure 1A). We analyzed the conserved domains of $12 \mathrm{NIGT1/HRS1/HHO}$ family genes in rice and A. thaliana. All NIGT1/HRS1/HHO proteins contain two conserved domains: Myb-DBD (C-terminal contains conserved SHLQKYR motif) and an HGD domain. HRS1, HHO2, HHO3, OsHHO3, and $\mathrm{OsHHO} 4$ have an EAR-like motif at the $\mathrm{N}$ terminal. HHO5 has two different EAR-like motifs at the $\mathrm{N}$ and $\mathrm{C}$ terminals (Figure 1B,C).

OsNIGT1 was found to be a transcribed inhibitory factor and could repress the transcription of downstream genes and itself. Further research showed that OsNIGT1 could bind to GAATC as a monomer or through a subunit of the dimer. OsNIGT1 also could bind to GAATATTC through the interaction of two subunits of the dimer. These two binding sites were also found in the promoters of some NIGT1/HRS1/HHO TFs genes in A. thaliana $[7,17]$. Therefore, the autorepression mechanism of OsNIGT1 may be conserved in monocotyledons and dicotyledons. This review further analyzed the binding sites of the promoter of all NIGT1/HRS1/HHO family genes in A. thaliana and rice (Figure 1D). The result indicated that the promoters of all NIGT1/HRS1/HHO family genes have the binding sites for autorepression in A. thaliana and rice. It is suggested that the NIGT1/HRS1/HHO TF family might have the autorepression mechanism universally.

\section{Function of NIGT1/HRS1/HHO TF Family the Absorption and Utilization of Nitrogen and Phosphorus in Plant}

Nitrogen $(\mathrm{N})$ and phosphorus $(\mathrm{P})$ are the key elements for plant growth and exist in many forms in nature. In organisms, $\mathrm{P}$ appears in the form of free phosphate ions, which are called inorganic phosphate (Pi). Because of the limited Pi content in the soil, plants are often in a Pi deficient state, which leads to a series of Pi starvation responses (PSR) [18,19]. The studies on the absorption and utilization of P by NIGT1/HRS1/HHO TFs are mainly concentrated in A. thaliana. Under Pi deficiency, the expression of HRS1 and HHO1 were increased in leaves and roots, suggesting that HRS1 and HHO1 played a role in PSR [6,20]. In addition, $\mathrm{HHO} 2$ can regulate its Pi homeostasis by Phosphate Starvation Response 1 protein (PHR1), which is the major regulator of PSR [21]. 
Nitrate is the main $\mathrm{N}$ source and the $\mathrm{N}$ signaling source for most land plants [7,22]. Some studies found that NIGT1/HRS1/HHO TFs arealso regulators of N starvation responses in A. thaliana and played an important role in $\mathrm{N}$ absorption and utilization. Under $\mathrm{N}$ starvation, the expression of NIGT1/HRS1/HHO TFs (i.e., HRS1, HHO1, HHO2, and $\mathrm{HHO} 3$ ) decrease rapidly, and the expression of $\mathrm{N}$ starvation response genes increase, which leads to $\mathrm{N}$ starvation responses. After $\mathrm{N}$ application, the expression of NIGT1/HRS1/HHO TFs (i.e., HRS1, HHO1, HHO2, and HHO3) is rapidly induced to repress the expression of $\mathrm{N}$ starvation gene responses, which reduce $\mathrm{N}$ starvation responses [23]. In addition, HRS1 and $\mathrm{HHO1}$ were found to be the early induced genes and major participants in nitrate signaling. The expression of HRS1 and HHO1 is regulated by the nitrate response transcription factors NIN-LIKE PROTEIN (NLP) and the nitrate transporters NRT1.1/NPF6.3 and NRT2.1 [10,24-26]. NIGT1/HRS1/HHO TFs also represses the expression of other genes related to $\mathrm{N}$ utilization, such as NITRATE REDUCTASE 1 (NIA1), GLUTAMINE SYNTHE-TASE 1;1 (GLN1;1) and GLN1;4. It is suggested that NIGT1/HRS1/HHO TFs can prevent the excessive accumulation of $\mathrm{N}$ by transducing the nitrogen saturation signal [27].

The balance of nitrogen and phosphorus is essential for the normal growth and development of plants. Studies have demonstrated that PHR1 can promote the expression of HRS1 by modulating PSR and reducing nitrate absorption. HRS1 and HHO1 can coordinate $\mathrm{N}$ and $\mathrm{P}$ by regulating the nitrate and phosphate starvation responses in $A$. thaliana $[10,26,28,29]$. Further study showed that HRS1, PHR1, and SPX (SYG1/PHO81/ XPR1, the inhibitory factor of PHR1) could form the NIGT1-SPX-PHR signaling pathway, which regulates the $\mathrm{N}$ content and coordinates the balance of $\mathrm{N}$ and $\mathrm{P}$ in response to PSR $[10,23,29]$. Moreover, the study showed that HHO5 and HHO6 were related to N and $\mathrm{P}$ responses and were the regulators of $\mathrm{N}$ absorption [30] (Figure 2A).

Similar to NIGT1/HRS1/HHO family genes in A. thaliana, the expression of OsNIGT1 can be rapidly and specifically induced by nitrate in rice. OsNIGT1 can also regulate nitrate utilization and can play critical roles in nitrate signaling in rice [7]. OsHHO3 and $\mathrm{OsHHO} 4$ are the key regulators in response to $\mathrm{N}$ deficiency and may play a role as the transcriptional inhibitors in $\mathrm{N}$ utilization [11,31]. However, the P absorption and utilization functions of NIGT1/HRS1/HHO TFs have yet to be reported in rice. In summary, NIGT1/HRS1/HHO TFs can regulate gene expression in the nitrate and phosphate signaling pathways and can coordinate nitrate and phosphate absorption. It is suggested that NIGT1/HRS1/HHO TFs play an important role in balancing the absorption and utilization of $\mathrm{N}$ and $\mathrm{P}$ in plants. 


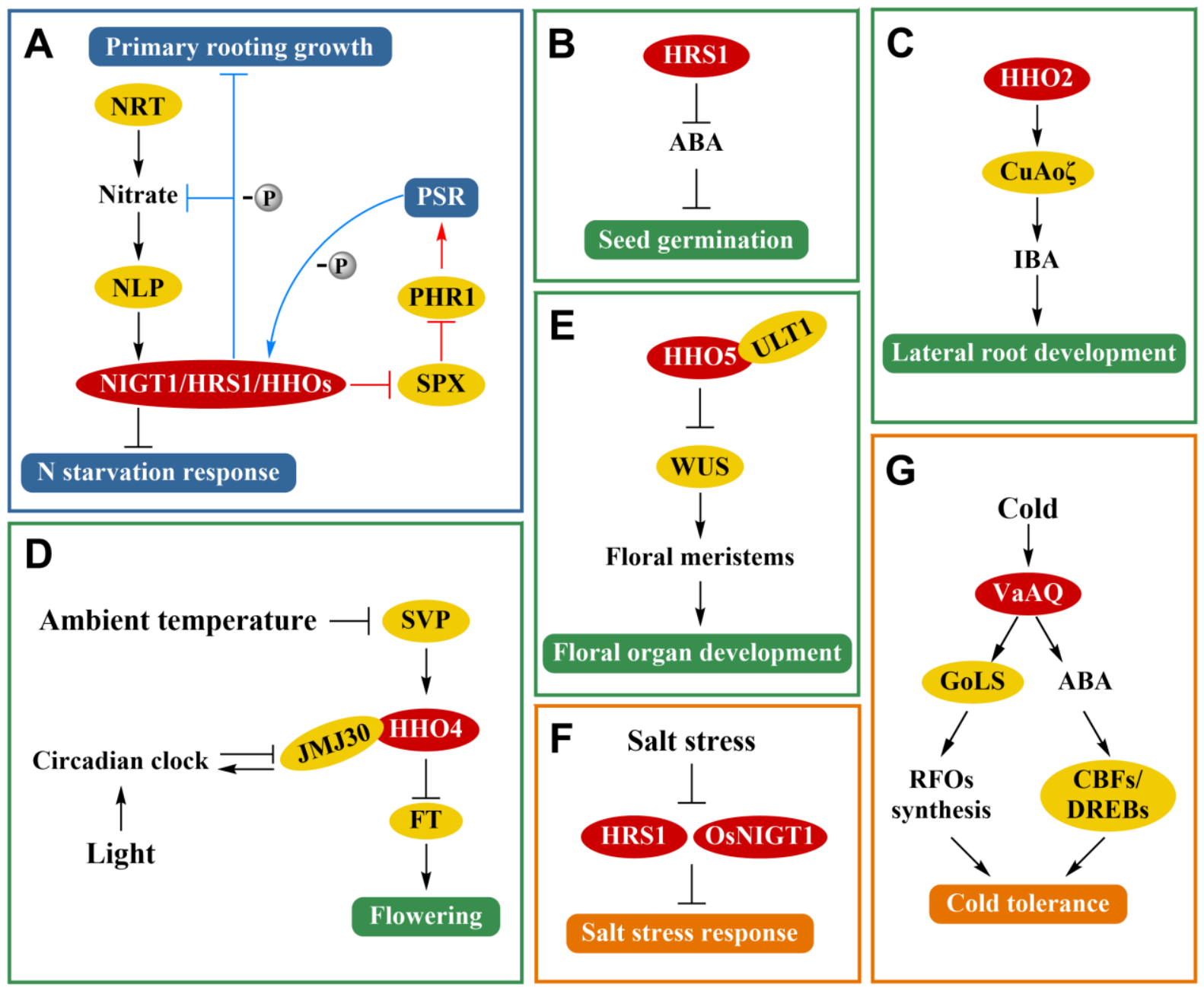

Figure 2. Simplified schematic of NIGT1/HRS1/HHO TFs involved in multifunctional regulation in plants. (A) The black arrow: Nitrate transporter NRT can increase the content of nitrate and can enhance the expression of the nitrate response transcription factors and induce the expression of NIGT1/HRS1/HHO family genes to inhibit the N starvation response. The red arrow: NIGT1/HRS1/HHO TFs can inhibit the expression of SPX and can induce the expression of PHR1 to promote PSR. The blue arrow: Under Pi deficiency, PSR can promote the expression of NIGT1/HRS1/HHO TFs to inhibit nitrate absorption and primary root growth. (B-E) Functions of NIGT1/HRS1/HHO TFs in plant growth and development. (B) HRS1 can promote seed germination by inhibiting ABA signaling. (C) $\mathrm{HHO} 2$ can increase the expression of $\mathrm{CuAO \zeta}$ and promotes IBA to enhance lateral root growth. (D) NIGT1/HRS1/HHO TFs are involved in flowering by coordinating temperature and light. SVP can promote the expression of $\mathrm{HHO}$. HHO4 can interact with JMJ30. Protein complex of $\mathrm{HHO} 4$ and JMJ30 repress the expression of FT gene to inhibit flowering. (E) HHO5 can interact with ULT1 and can inhibit the expression of WUS to participate in the homeostasis of floral meristems and the growth and development of floral organs. $(\mathbf{F}, \mathbf{G})$ Functions of NIGT1/HRS1/HHO TFs in abiotic stress. (F) The expression of HRS1 and OsNIGT1 are repressed under salt stress. Mutants of HRS1 and OsNIGT1 can enhance tolerance to salt stress. (G) VaAQ can improve low temperature tolerance. VaAQ increased the synthesis of RFOs by increasing GoLS activity to improve low temperature tolerance. VaAQ also regulates the expression of the $C B F S$ or $D R E B s$ and improves tolerance to low temperature stress through the ABA pathway. Promoting and repressive effects are indicated by $\rightarrow$ and $\dashv$, respectively, and P deficiency is denoted by $-\mathrm{P}$.

\section{Functions of NIGT1/HRS1/HHO TF Family in Plant Growth and Development}

Evidence suggests that NIGT1/HRS1/HHO TFs not only participate in the absorption and utilization of $\mathrm{N}$ and $\mathrm{P}$, but also show complex regulatory mechanisms and networks involved in seed germination, root development, and flowering. An overexpression of HRS1 could inhibit the elongation of primary roots in the absence of Pi. HHO1 could also inhibit the growth of primary roots under a specific ratio of $\mathrm{N}$ and $\mathrm{P}$. The double 
knockout mutants of HRS1 and HHO1 significantly inhibit the growth of primary roots under nitrate only treatment $[6,28]$ (Figure 2A). In addition, HRS1 is a negative regulator of ABA signaling and can promote seed germination by inhibiting ABA signaling in $A$. thaliana. HRS1 can enhance $\mathrm{H}^{+}$-ATPase activity on the cell membrane and the elongation of cells in the hypocotyl and hypocotyl-radicle transition zone to promote seed germination in A. thaliana [32] (Figure 2B). In addition to being related to primary roots growth, NIGT1/HRS1/HHO TFs can also promote the development of lateral roots. The copper amine oxidase $\zeta(\mathrm{CuAO} \zeta)$ is located in peroxisomes in $A$. thaliana. $\mathrm{CuAO} \zeta$-derived ROS is involved in the development of lateral roots induced by indole-3-butyric acid (IBA) [33]. $\mathrm{HHO} 2$ can increase the expression of $\mathrm{CuAO} \zeta$ and can enhance lateral root growth $[21,34]$ (Figure 2C).

A recent study showed that $\mathrm{HHO} 4$ and $\mathrm{HHO} 5$ were associated with flowering. The short vegetative phase (SVP) TF is affected by temperature to regulate flowering. SVP can promote the expression of $\mathrm{HHO} 4$ in A. thaliana. Meanwhile, HHO4 can interact with JMJ30, which is the $\mathrm{H} 3 \mathrm{~K} 36 \mathrm{Me} 2$ demethylase and is involved in light-responsive circadian clock. The protein complexes of HHO4 and JMJ30 repress the expression of the FLOWERING LOCUS T (FT) gene to inhibit flowering. It is suggested that NIGT1/HRS1/HHO TFs are involved in flowering by coordinating temperature and light [8] (Figure 2D). Ultrapetala1 (ULT1) is a key negative regulator of stem cell activity in shoot apical meristems and floral meristems. HHO5 can interact with ULT1 and the inhibited expression of the central transcription factor WUSCHEL (WUS), which controls the balance of shoot apical meristems in apical meristems. HHO5 and ULT1 can participate in the homeostasis of floral meristems and the growth and development of floral organs [9] (Figure 2E). In conclusion, NIGT1/HRS1/HHO TFs may be involved in seed germination, root development, and flowering by responding to light, temperature, and hormone (ABA and IBA) signals. Therefore, NIGT1/HRS1/HHO TFs play an important role in plant growth and development.

\section{Function of NIGT1/HRS1/HHO TF Family in Plant Abiotic Stress}

Up until now, few studies have been reported on the relationship between NIGT1/ HRS1/HHO TFs and abiotic stress. Some studies have found that NIGT1/HRS1/HHO TFs may be related to salt and low temperature stress. The expression of HRS1 was repressed under salt stress in $A$. thaliana [35]. Further research has demonstrated that the membrane ion leakages of the HRS1 mutant and OsNIGT1 mutant significantly decreases in A. thaliana and rice, respectively, and the HRS1 mutant and OsNIGT1 mutant could continue to grow and fruit in $A$. thaliana and rice after salt stress. It is suggested that the NIGT1/HRS1/HHO TFs are related to salt tolerance [35] (Figure 2F). $V a A Q$, a grape TF gene homologous to $H R S 1, H H O 2$, and $H H O 3$, can improve the low temperature tolerance in transgenic $A$. thaliana and transgenic $V$. amurensis. VaAQ increases the synthesis of raffinose family oligosaccharides (RFOs) by increasing the activity of galactinol synthase (GoLS) to improve low temperature tolerance. Through the ABA pathway, VaAQ also regulates the expression of $C B F s$ or DREBs, which are the core genes of low-temperature signaling, and improves tolerance to low temperature stress [12] (Figure 2G).

\section{Conclusions}

The NIGT1/HRS1/HHO TF family is a new family that was first named in 2009. The NIGT1/HRS1/HHO TF family has seven members (HRS1 and HHO1-HHO6) in A. thaliana and five members (OsNIGT1 and OsHHO1-OsHHO4) in rice. NIGT1/HRS1/HHO TFs play an important coordinating and regulating role of $\mathrm{N}$ and $\mathrm{P}$ absorption and utilization, growth and development, and abiotic stress. The coordinated regulation mechanism of plant nutrition and development is essential to cope with the ever-changing environment [3]. Roots play a key role in the adaptive responses mediated by Pi deficiency. Meanwhile, Pi affects the growth of primary roots $[36,37]$. Nitrates are related to the growth of lateral roots and can counteract the effect of glutamate on the growth of primary 
roots [38]. However, the mechanism by which plants integrate these key nutrients to regulate development is still unknown. NIGT1/HRS1/HHO TFs have been shown to coordinate the absorption and utilization of $\mathrm{N}$ and $\mathrm{P}$ and to participate in root development regulated by $\mathrm{N}$ and $\mathrm{P}$ through unknown mechanisms. Therefore, NIGT1/HRS1/HHO TFs are the key to understanding the relationship between the $\mathrm{N}$ and $\mathrm{P}$ signaling pathways and root development. In addition, previous studies on NIGT1/HRS1/HHO TFs have mainly focused on the utilization of N and P. The role of NIGT1/HRS1/HHO TFs in plant growth and abiotic stress has not been studied in depth. Currently, NIGT1/HRS1/HHO TFs have been found to regulate seed germination and to enhance low-temperature stress tolerance through the ABA signaling pathway. These studies also provide references for further research on the molecular mechanism between NIGT1/HRS1/HHO TFs and ABA signals. Moreover, whether NIGT1/HRS1/HHO TFs are also involved in the synthesis or signaling pathways of other plant hormones remains to be further explored. In conclusion, although many questions remain unanswered, further research will expand our understanding of the function of NIGT1/HRS1/HHO TFs in plant growth, development, and stress responses.

Author Contributions: Y.G. and Q.L. conceived and designed the main content; Q.L., L.Z., and D.Z. wrote the manuscript with help from Y.L. All authors have read and agreed to the published version of the manuscript.

Funding: This work was supported by the National Natural Science Foundation of China (No. 31771686); the Natural Science Foundation of Jiangsu Province (BK20181216); the modern agriculture project of Yangzhou city (YZ2019035).

Institutional Review Board Statement: Not applicable.

Informed Consent Statement: Not applicable.

Data Availability Statement: Not applicable.

Acknowledgments: We thank the Talent Project of Yangzhou University and the Priority Academic Program Development of Jiangsu Higher Education Institutions.

Conflicts of Interest: The authors declare no conflict of interest.

\section{References}

1. Riechmann, J.L.; Heard, J.; Martin, G.; Reuber, L.; Jiang, C.Z.; Keddie, J.; Adam, L.; Pineda, O.; Ratcliffe, O.J.; Samaha, R.R.; et al. Arabidopsis transcription factors: Genome-wide comparative analysis among eukaryotes. Science 2000, 290, 2105-2110. [CrossRef]

2. Hosoda, K.; Imamura, A.; Katoh, E.; Hatta, T.; Tachiki, M.; Yamada, H.; Mizuno, T.; Yamazaki, T. Molecular structure of the GARP family of plant Myb-related DNA binding motifs of the Arabidopsis response regulators. Plant Cell 2002, 14, 2015-2029. [CrossRef]

3. Safi, A.; Medici, A.; Szponarski, W.; Ruffel, S.; Lacombe, B.; Krouk, G. The world according to GARP transcription factors. Curr. Opin. Plant Biol. 2017, 39, 159-167.

4. Jenkins, M.T. A Second Gene Producing Golden Plant Color in Maize. Am. Nat. 1926, 60, 484-488. [CrossRef]

5. Hall, L.N.; Rossini, L.; Cribb, L.; Langdale, J.A. GOLDEN 2: A Novel Transcriptional Regulator of Cellular Differentiation in the Maize Leaf. Plant Cell 1998, 10, 925-936. [CrossRef]

6. Liu, H.; Yang, H.X.; Wu, C.M.; Feng, J.J.; Liu, X.; Qin, H.J.; Wang, D.W. Overexpressing HRS1 Confers Hypersensitivity to Low Phosphate-elicited Inhibition of Primary Root Growth in Arabidopsis thaliana. J. Integr. Plant Biol. 2009, 51, 382-392. [CrossRef] [PubMed]

7. Sawaki, N.; Tsujimoto, R.; Shigyo, M.; Konishi, M.; Toki, S.; Fujiwara, T.; Yanagisawa, S. A Nitrate-Inducible GARP Family Gene Encodes an Auto-Repressible Transcriptional Repressor in rice. Plant Cell Physiol. 2013, 54, 506-517. [CrossRef]

8. Yan, Y.Y.; Shen, L.S.; Chen, Y.; Bao, S.J.; Thong, Z.H.; Yu, H. A MYB-domain Protein EFM Mediates Flowering Responses to Environmental Cues in Arabidopsis. Dev. Cell 2014, 30, 437-448. [CrossRef] [PubMed]

9. Moreau, F.; Thevenon, E.; Blanvillain, R.; Lopez-Vidriero, I.; Franco-Zorrilla, J.M.; Dumas, R.; Parcy, F.; Morel, P.; Trehin, C.; Carles, C.C. The Myb-domain protein ULTRAPETALA1 INTERACTING FACTOR 1 controls floral meristem activities in Arabidopsis. Development 2016, 143, 1108-1119.

10. Maeda, Y.; Konishi, M.; Kiba, T.; Sakuraba, Y.; Sawaki, N.; Kurai, T.; Ueda, Y.; Sakakibara, H.; Yanagisawa, S. A NIGT1-centred transcriptional cascade regulates nitrate signalling and incorporates phosphorus starvation signals in Arabidopsis. Nat. Commun. 2018, 9, 1376. [CrossRef] 
11. Ueda, Y.; Ohtsuki, N.; Kadota, K.; Tezuka, A.; Nagano, A.J.; Kadowaki, T.; Kim, Y.; Miyao, M.; Yanagisawa, S. Gene regulatory network and its constituent transcription factors that control nitrogen-deficiency responses in rice. New Phytol. 2020, 227, 1434-1452. [CrossRef] [PubMed]

12. Sun, X.M.; Matus, J.T.; Wong, D.C.J.; Wang, Z.M.; Chai, F.M.; Zhang, L.L.; Fang, T.; Zhao, L.; Wang, Y.; Han, Y.P.; et al. The GARP/MYB-related grape transcription factor AQUILO improves cold tolerance and promotes the accumulation of raffinose family oligosaccharides. J. Exp. Bot. 2018, 69, 1749-1764. [CrossRef]

13. Massari, M.E.; Murre, C. Helix-Loop-Helix Proteins: Regulators of Transcription in Eucaryotic Organisms. Mol. Cell. Biol. 2000, 20, 429-440. [CrossRef] [PubMed]

14. Rossini, L.; Cribb, L.; Martin, D.J.; Langdale, J.A. The Maize Golden2 Gene Defines a Novel Class of Transcriptional Regulators in Plants. Plant Cell 2001, 13, 1231-1244. [CrossRef] [PubMed]

15. Tiwari, S.B.; Hagen, G.; Guilfoyle, T.J. Aux/IAA Proteins Contain a Potent Transcriptional Repression Domain. Plant Cell 2004, 16, 533-543. [CrossRef]

16. Wang, W.; Wang, X.T.; Wang, Y.T.; Zhou, G.H.; Wang, C.; Hussain, S.D.; Adnan Lin, R.; Wang, T.Y.; Wang, S.C. SlEAD1, an EAR motif-containing ABA down-regulated novel transcription repressor regulates ABA response in tomato. GM Crops Food 2020, 11, 275-289. [CrossRef]

17. Yanagisawa, S. Characterization of a nitrate-inducible transcriptional repressor NIGT1 provides new insights into DNA recognition by the GARP family proteins. Plant Signal. Behav. 2013, 8, e24447. [CrossRef]

18. Ticconi, C.A.; Abel, S. Short on phosphate: Plant surveillance and countermeasures. Trends Plant Sci. 2004, 9, 548-555. [CrossRef]

19. Wu, P.; Shou, H.X.; Xu, G.H.; Lian, X.M. Improvement of phosphorus efficiency in rice on the basis of understanding phosphate signaling and homeostasis. Curr. Opin. Plant Biol. 2013, 16, 205-212. [CrossRef]

20. Misson, J.; Raghothama, K.G.; Jain, A.; Jouhet, J.; Block, M.A.; Bligny, R.; Ortet, P.; Creff, A.; Somerville, S.; Rolland, N.; et al. A genome-wide transcriptional analysis using Arabidopsis thaliana Affymetrix gene chips determined plant responses to phosphate deprivation. Proc. Natl. Acad. Sci. USA 2005, 102, 11934-11939. [CrossRef]

21. Nagarajan, V.K.; Satheesh, V.; Poling, M.D.; Raghothama, K.G.; Jain, A. Arabidopsis MYB-Related HHO2 Exerts a Regulatory Influence on a Subset of Root Traits and Genes Governing Phosphate Homeostasis. Plant Cell Physiol. 2016, 57, 1142-1152. [CrossRef]

22. Crawford, N.M.; Forde, B.G. Molecular and developmental biology of inorganic nitrogen nutrition. Arab. Book 2002, 1, e0011. [CrossRef] [PubMed]

23. Kiba, T.; Inaba, J.; Kudo, T.; Ueda, N.; Konishi, M.; Mitsuda, N.; Takiguchi, Y.; Kondou, Y.; Yoshizumi, T.; Ohme-Takagi, M.; et al Repression of Nitrogen Starvation Responses by Members of the Arabidopsis GARP-Type Transcription Factor NIGT1/HRS1 Subfamily. Plant Cell 2018, 30, 925-945. [CrossRef]

24. Konishi, M.; Yanagisawa, S. Arabidopsis NIN-like transcription factors have a central role in nitrate signalling. Nat. Commun. 2013, 4, 1617. [CrossRef] [PubMed]

25. Marchive, C.; Roudier, F.; Castaings, L.; Brehaut, V.; Blondet, E.; Colot, V.; Meyer, C.; Krapp, A. Nuclear retention of the transcription factor NLP7 orchestrates the early response to nitrate in plants. Nat. Commun. 2013, 4, 1713. [CrossRef]

26. Menz, J.; Li, Z.; Schulze, W.X.; Ludewig, U. Early nitrogen-deprivation responses in Arabidopsis roots reveal distinct differences on transcriptome and (phospho-) proteome levels between nitrate and ammonium nutrition. Plant J. 2016, 88, 717-734. [CrossRef]

27. Ueda, Y.; Yanagisawa, S. Perception, transduction, and integration of nitrogen and phosphorus nutritional signals in the transcriptional regulatory network in plants. J. Exp. Bot. 2019, 70, 3709-3717. [CrossRef] [PubMed]

28. Medici, A.; Marshall-Colon, A.; Ronzier, E.; Szponarski, W.; Wang, R.C.; Gojon, A.; Crawford, N.M.; Ruffel, S.; Coruzzi, G.M.; Krouk, G. AtNIGT1/HRS1 integrates nitrate and phosphate signals at the Arabidopsis root tip. Nat. Commun. 2015, 6, 6274. [CrossRef]

29. Ueda, Y.; Kiba, T.; Yanagisawa, S. Nitrate-inducible NIGT1 proteins modulate phosphate uptake and starvation signalling via transcriptional regulation of SPX genes. Plant J. 2020, 102, 448-466. [CrossRef]

30. Varala, K.; Marshall-Colon, A.; Cirrone, J.; Brooks, M.D.; Pasquino, A.V.; Leran, S.; Mittal, S.; Rock, T.M.; Edwards, M.B.; Kim, G.J.; et al. Temporal transcriptional logic of dynamic regulatory networks underlying nitrogen signaling and use in plants. Proc. Natl. Acad. Sci. USA 2018, 115, 6494-6499. [CrossRef]

31. Obertello, M.; Shrivastava, S.; Katari, M.S.; Coruzzi, G.M. Cross-Species Network Analysis Uncovers Conserved NitrogenRegulated Network Modules in Rice. Plant Physiol. 2015, 168, 1830-1843. [CrossRef] [PubMed]

32. Wu, C.M.; Feng, J.J.; Wang, R.; Liu, H.; Yang, H.X.; Rodriguez, P.L.; Qin, H.J.; Liu, X.; Wang, D.W. HRS1 acts as a negative regulator of abscisic acid signaling to promote timely germination of Arabidopsis seeds. PLoS ONE 2012, 7, e35764. [CrossRef]

33. Qu, Y.; Wang, Q.; Guo, J.; Wang, P.; Song, P.; Jia, Q.; Zhang, X.; Kudla, J.; Zhang, W.; Zhang, Q. Peroxisomal CuAO $\zeta$ and its product $\mathrm{H} 2 \mathrm{O} 2$ regulate the distribution of auxin and IBA-dependent lateral root development in Arabidopsis. J. Exp. Bot. 2017, 68, 4851-4867. [CrossRef] [PubMed]

34. Qu, Y.; Liu, X.; Zhang, X.D.; Tang, Y.W.; Hu, Y.W.; Chen, S.Y.; Xiang, L.X.; Zhang, Q. Transcriptional regulation of Arabidopsis copper amine oxidase $\zeta(\mathrm{CuAO} \zeta)$ in indole-3-butyric acid-induced lateral root development. Plant Growth Regul. 2019, 89, 287-297. [CrossRef]

35. Mito, T.; Seki, M.; Shinozaki, K.; Ohme-Takagi, M.; Matsui, K. Generation of chimeric repressors that confer salt tolerance in Arabidopsis and rice. Plant Biotechnol. J. 2011, 9, 736-746. [CrossRef] [PubMed] 
36. Williamson, L.C.; Ribrioux, S.P.C.P.; Fitter, A.H.; Leyser, H.M.O. Phosphate availability regulates root system architecture in Arabidopsis. Plant Physiol. 2001, 126, 875-882. [CrossRef] [PubMed]

37. Gruber, B.D.; Giehl, R.F.H.; Friedel, S.; Von Wiren, N. Plasticity of the Arabidopsis root system under nutrient deficiencies. Plant Physiol. 2013, 163, 161-179. [CrossRef] [PubMed]

38. Vidal, E.A.; Tamayo, K.P.; Gutierrez, R.A. Gene networks for nitrogen sensing, signaling, and response in Arabidopsis thaliana. Wiley Interdiscip. Rev. Syst. Biol. Med. 2010, 2, 683-693. 